\title{
Multifrequency Behaviour of Polars
}

\author{
K. Reinsch ${ }^{1}$ \\ ${ }^{1}$ Georg-August-Universität Göttingen, Institut für Astrophysik, Friedrich-Hund-Platz 1, 370r7 Göttingen, Germany \\ Corresponding author: reinsch@astro.physik.uni-goettingen.de
}

\begin{abstract}
Cataclysmic variables emit over a wide range of the electromagnetic spectrum. In this paper I will review observations of polars in relevant passbands obtained during the last decade and will discuss their diagnostical potential to access the physics of the main components within the binary systems. This will include a discussion of intrinsic source variability and the quest for simultaneous multi-frequency observations.
\end{abstract}

Keywords: cataclysmic variables - polars - optical - spectroscopy - photometry - IR - UV - X-rays.

\section{Introduction}

In the standard model of magnetic cataclysmic variables a polar is described as a semi-detached binary in which matter from a Roche-lobe filling late-type mainsequence star is coupled outside the circularization radius to the field lines of a highly-magnetic white dwarf and channeled onto small accretion region(s) in the vicinity of the magnetic pole(s). The system components can be observationally disentangled - at least to some degree - by their characteristic emission in different wavelength regimes (see Fig. 1). The contribution of the binary components to the spectral energy distribution of the system will be discussed individually and in the context of multi-frequency observations. The basic understanding of polars has not much changed since the comprehensive review by Warner (1995). I will, therefore, concentrate on recent observational findings which led to a more detailed understanding of the physical processes involved.

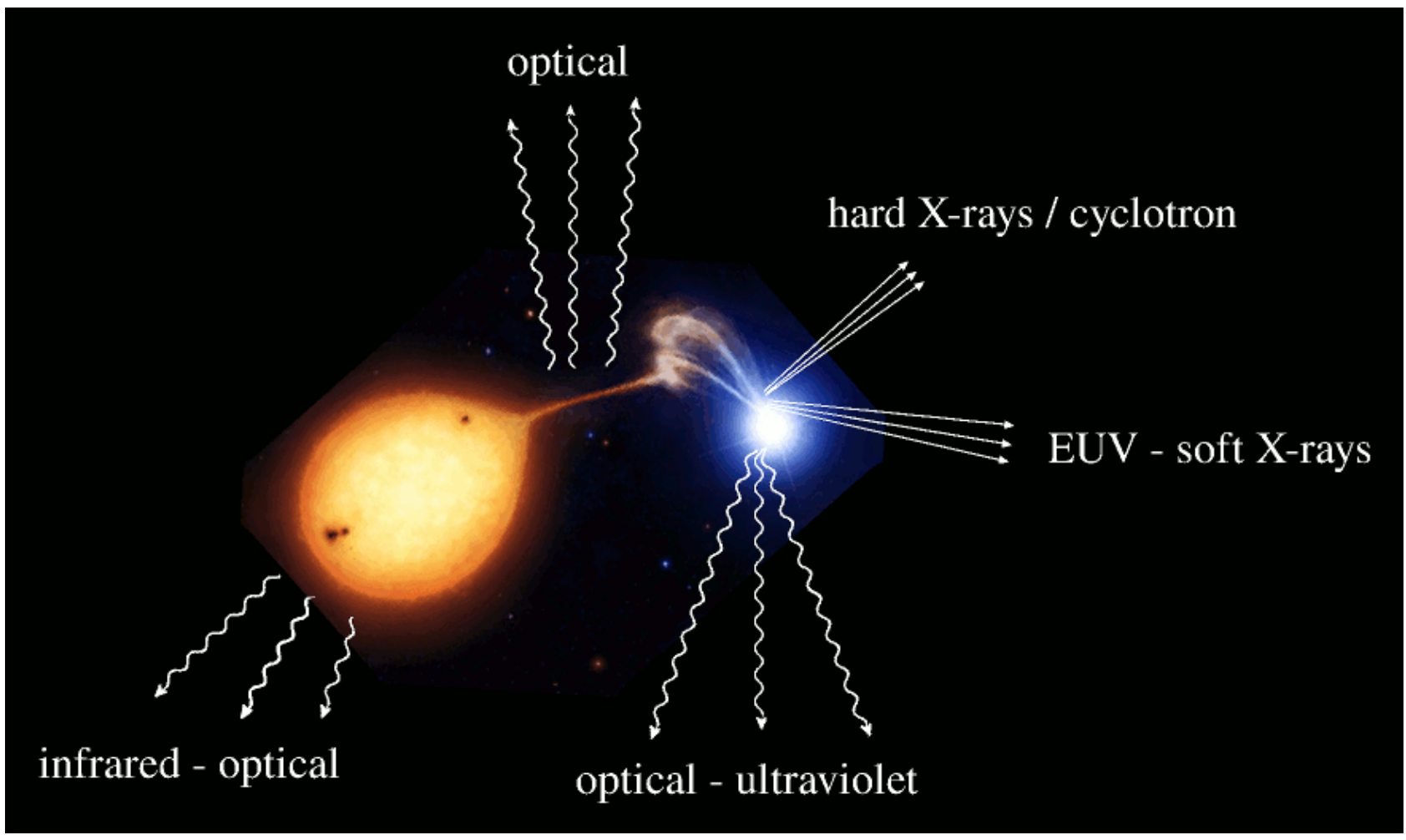

Figure 1: Schematic view of a polar and main emission regions (Iris Traulsen, adapted from Marc Garlick). 


\section{Binary Components and Spectral Energy Distribution}

\subsection{Secondaries in polars}

Secondaries in polars emit most of their light in the red and near IR. But even in these bands their luminosity is generally outshone by other components within the binary. The secondaries are, therefore, best studied when the system happens to be in a low state of accretion or during eclipses of the accretion region and the white dwarf. Optical and near-IR studies have revealed that their spectra are consistent with normal late-type dwarfs (or brown dwarfs). Contrary to similar studies of non-magnetic systems, no significant abundance anomalies have been found from near-IR spectroscopy of the secondaries in polars. This provides evidence that magnetic and non-magnetic systems might take different evolutionary paths (Harrison et al., 2005).

Spectroscopy during the eclipse of the primary combined with phase-resolved near-IR photometry of polars can be used to determine the spectral type and the brightness of the secondary, as has been shown e.g. for the long-period polar V1309 Ori (Reinsch at al., 2006). Using calibrations of the surface brightness of M-dwarfs (e.g. Beuermann 2000), from the brightness of the secondary an accurate distance of the system can be derived.

\subsection{Circumbinary dust disk}

Mid-infrared observations of the short-period polar EF Eri with the Infrared Spectrograph (IRS) on the Spitzer space telescope have revealed the presence of emission in the $3-14 \mu \mathrm{m}$ wavelength range which exceeds the combined spectral energy distribution of the white dwarf, the L5-type brown dwarf secondary, and cyclotron radiation from the $B \approx 13 \mathrm{MG}$ white dwarf magnetic field. Hoard et al. (2007) concluded that the spectrum longward of $\sim 5 \mu \mathrm{m}$ is dominated by emission from a circumbinary dust disk. In a later paper, however, Harrison et al. (2013) argue based on combined ground-based, Spitzer, WISE, and Herschel observation of the two polars AM Her and EF Eri that their midinfrared excess can be fully explained by optically thin emission from the lowest cyclotron harmonics $(n \leq 3)$.

\subsection{Stellar activity of the secondary}

From long-term observations of polars it is well known that most systems alternate between low and high states and sometimes also intermediate states of accretion. The best studied system is AM Her with a record of 33 years of optical observations by the American Association of Variable Star Observers (AAVSO, http://www.aavso.org/). Due to the absence of an ac- cretion disk in polars such changes must reflect the immediate response of the system on variations of the mass-transfer rate. Stellar activity of the donor star has been proposed as a possible mechanism to induce such variations (e.g. Hessman et al., 2000). In a recent analysis of short- and long-term brightness variations of five polars, periodic variations were indicated which could result from magnetic cycles in the secondary stars (Kalomeni, 2012).

Kafka et al. (2010) present another piece of evidence for stellar activity from phase-resolved high-resolution spectroscopy of polars in low states. They interprete two bright satellites of the central low-velocity component in the $\mathrm{H} \alpha$ Doppler map of BL Hyi as prominencelike magnetic loops kept in place by magnetic field interactions between the white dwarf and the donor star.

Howell et al. (2006) have monitored the long-term variation of the $\mathrm{H} \alpha$ equivalent width of $\mathrm{EF}$ Eri during its extended low state and attribute the observed behaviour to chromospheric activity of the secondary. In addition they identify magnetic field interaction between the two stars as a probable mechanism that would concentrate stellar activity on the white dwarf facing hemisphere of the secondary.

\subsection{Coupling region}

Partially ionized matter released from the secondary over the L1 point is assumed to follow initially a freefalling trajectory. At the magnetospheric radius of the white dwarf the ram pressure of the infalling gas is balanced by the magnetic pressure and the accretion stream couples to the magnetic field of the white dwarf. Observationally little information is available on the processes occurring in this coupling region.

XMM-Newton observations of V1309 Ori show a prolonged ingress of the soft X-ray light curve with Xray emission being visible beyond the geometrical occultation of the white dwarf. Schwarz et al. (2005) have suggested plasma heated by shocks in the coupling region as a possible origin of the X-ray emission seen during eclipse.

\subsection{Accretion curtain}

Beyond the coupling region accretion becomes magnetically controlled and the initially narrow ballistic stream is diverted off the orbital plane and forms a broad curtain-like structure through which matter is channeled into the vicinity of the magnetic poles of the white dwarf. The accretion stream and curtain are characterized by an optical and UV emission-line spectrum comprising strong lines of neutral hydrogen and of ionized helium. Using relatively simple geometrical assumptions indirect imaging techniques, like Doppler 
tomography, Roche tomography, accretion stream mapping, and eclipse mapping, have been well established to confine the geometrical parameters of different parts of the accretion stream (e.g. Schwope et al., 2004).

\subsection{White dwarf}

White dwarfs in polars have typical temperatures in the range 10,000-15,000 K. Their photospheric emission has been well studied in the UV and far-UV spectra of polars in low states of accretion. Based on a far-UV spectroscopic survey with HST/ STIS, Araujo-Betancor et al. (2005) found that at any given orbital period white dwarfs in magnetic CVs are colder than those in non magnetic CVs with the differences in effective temperatures being largest for systems above the period gap. As a possible explanation they suggest that magnetic braking in polars might be reduced.

The magnetic field structure of several white dwarfs in polars has been studied in detail using phase-resolved circular spectropolarimetry and Zeeman tomography (e.g. Beuermann et al., 2007 and references therein). In most cases the field structure departs significantly from that of a dipole field and higher order multipoles are required to adequately describe the observed Zeeman spectra. Accreting material is flowing preferentially along field lines reaching far out towards the ballistic part of the stream. Depending on field geometry and accretion rate, this leads to one or more accretion regions on the white dwarf.

\subsection{Accretion region}

In the standard scenario actual accretion onto magnetic white dwarfs occurs in a comparably small region of some $0.1 \%$ of the white dwarf surface. Infalling matter forms an accretion column with a shock front at some height above the white dwarf where the supersonic gas of the accretion stream is decelerated to a subsonic settling flow. The shock height and the depression of the post-shock flow below the photosphere depends on the magnetic field strength, the mass of the white dwarf, and the specific accretion rate. The structure of the accretion column has been studied theoretically. The emergent spectra have been calculated by radiationhydrodynamical models as a function of mass-flow rate and field strength (e.g. Fischer \& Beuermann, 2001; Beuermann, 2004). At low mass-flow rates, the shock stands above the atmosphere and Bremsstrahlung radiation is the main cooling process. For larger accretion rates the emission is hidden behind the column density and reprocessed as soft X-rays.

Irradiation is the principle cause for a large heated accretion cap extending far beyond the impact region of the accreted material. Calculations with a standard LTE stellar atmosphere code including an angledependent external radiation source have demonstrated that much of the reprocessed light appears in far UV and not at soft X-rays (König et al., 2006).

Observationally the temperature distribution in the accretion spot could be demonstrated by the analysis of the Chandra LETG spectrum of AM Her. This data provide enough photons to show that the soft X-ray component cannot be fitted by a single temperature model (Beuermann et al., 2012).

At high mass-transfer rates sufficiently dense blobs can penetrate to large optical depths in the white-dwarf atmosphere submerging the shock below the photosphere. The most extreme example of this "blobby accretion" case has been observed in V1309 Ori. Its soft X-ray light curve obtained with XMM-Newton shows X-ray emission occuring in flares of typically $10 \mathrm{~s}$ time scale. This corresponds to a length scale of $4 \times 10^{9} \mathrm{~cm}$ for the infalling filaments (Schwarz et al., 2005).

Short-term spectral variability at X-ray wavelengths could be studied in the ROSAT PSPC high state observation of AM Her which contains 1.3 million counts. Significant variability on time scales down to $200 \mathrm{~ms}$ could be detected. Correlated spectral and count-rate variations are seen in flares on time scales down to $1 \mathrm{~s}$, demonstrating the heating and cooling associated with individual accretion events (Beuermann et al., 2008).

Some polars are even bright enough that X-ray emission line spectroscopy is feasible with current instruments. The high-diagnostics potential of such observations has been demonstrated e.g. by Girich et al. (2007), Mauche et al. (2003), Mauche et al. (2009).

\section{$3 \quad$ Variability of Spectral Energy Distribution}

Several polars have been studied at different epochs yielding that their optical, UV, and X-ray fluxes can vary by more than an order of magnitude. As an example, Fig. 2 shows the integrated energy flux $\nu f_{\nu}$ from the infrared to the hard X-ray regime of the polar RX J1007.5-2017 collected with various instruments between 1992 and 2001. The left panel shows spectrophotometry of 1992, 1997, and 2000 (black curves), supplemented by the 2MASS J, H, and K-band fluxes of 1999 (red filled squares) and the visual and ultraviolet fluxes measured with the optical monitor on board of XMM-Newton simultaneous to the 2001 X-ray observation (red filled circles). The green curve represents the adjusted flux distribution of the dM3 star LHS58 which is expected to match the spectral type of the donor star and to be the main contribution to the 1997 low-state spectrum. 

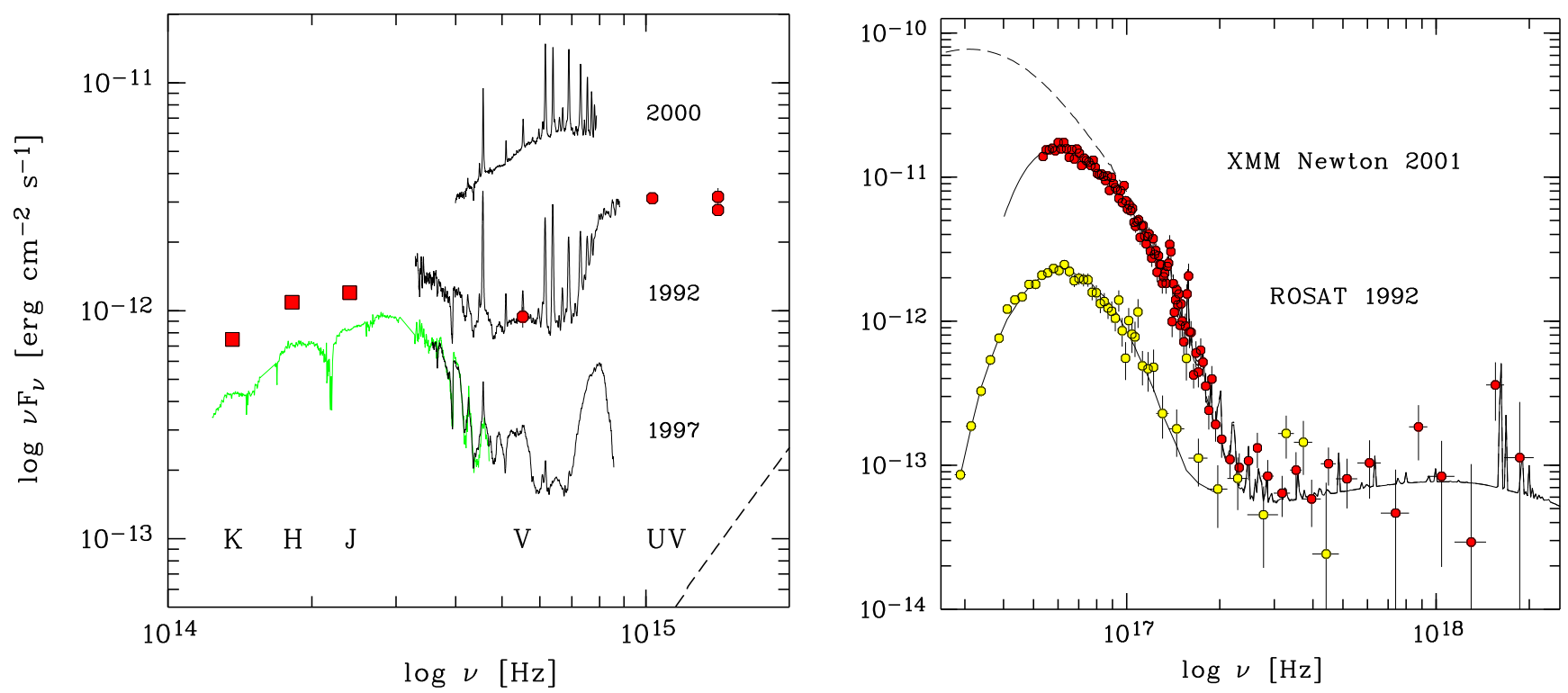

Figure 2: Spectral energy distribution of the polar RXJ1007.5-2017 at different epochs (from: Thomas et al. 2012)

The right panel shows the incident spectra for the 2001 XMM pn observation (red filled circles) and the 1992 ROSAT PSPC observation (open circles). The dashed curve illustrates the "source spectrum" corrected for interstellar absorption (Thomas et al., 2012).

\section{Conclusions}

X-ray and optical surveys, like the ROSAT all-sky survey and the Sloan Digital Sky Survey, let to the discovery of a large number of new polars during the recent two decades increasing the sample size to 135 polars with known orbital periods (Ritter \& Kolb 2003; 2013).

In addition, sensitivity and instrumental capabilities at all wavelengths have been significantly enhanced. Taking both together, polars provide an important lab to quantitatively understand individual systems and physical processes of matter under extreme conditions. In this sense, we are indeed currently in a "golden age" of research on polars.

There are still several fundamental questions remaining open. Among these are: Do we fully understand the evolution of polars? What drives accretionrate variations? Do polars with fields as high as in single white dwarfs exist? What is the origin of the magnetic field in polars? What exactly happens in the coupling region?

Multi-frequency observations are a powerful tool to improve our understanding of polars in future. Due to large variations of the accretion rate on different time scales simultaneous coverage at different wavelengths is mandatory.

\section{Acknowledgement}

I thank the many people who contributed to increase our knowledge on polars over the last decade, and kindly acknowledge fruitful and long-lasting collaborations with Klaus Beuermann, Vadim Burwitz, Essam El-Kholy, Fabian Euchner, Boris Gänsicke, Yonggi Kim, Axel Schwope, Robert Schwarz, Hans-Christoph Thomas, Iris Traulsen, and Fred Walter which led to several papers I have referred to in this contribution.

\section{References}

[1] Araujo-Betancor, S., Gänsicke, B.T., Long, K.S., Beuermann, K., de Martino, D., Sion, E.M., Szkody, P., 2005, ApJ 622, 589 doi:10.1086/427914

[2] Beuermann, K., 2000, New Astr. Rev. 44, 93 doi:10.1016/S1387-6473(00)00020-8

[3] Beuermann, K., 2004, ASPC 315, 187

[4] Beuermann, K., El Kholy, E., Reinsch, K., 2008, A\&A 481, 771

[5] Beuermann, K., Euchner, F., Reinsch, K., Gänsicke, B.T., 2007, A\&A 463, 647

[6] Beuermann, K., Burwitz, V., Reinsch, K., 2012, A\&A 543A, 41

[7] Fischer, A., Beuermann, K., 2001, A\&A 373, 211; 
[8] Girich, V., Rana, V.R., Singh, K.P., 2007, ApJ 658,525

[9] Harrison, T.E., Howell, S.B., Szkody, P., Cordova, F.A., 2005, ApJ 632, L123 doi:10.1086/498067

[10] Harrison, T.E., Hamilton, R.T., Tappert, C., Hoffman, D.I., Campbell, R.K., 2013, AJ 145, 19.

[11] Hessman, F., Gänsicke, B., Mattei, J., 2000, A\&A 361,952

[12] Hoard, D.W., Howell, S.B., Brinkworth, C.S., Ciardi, D.R., Wachter, S., 2007, ApJ 671, 734. doi:10.1086/522694

[13] Howell, S.B., Walter, F.M., Harrison, T.E., Huber, M.E., Becker, R.H., White, R.L., 2006, ApJ 652, 709 doi:10.1086/507603

[14] Kafka, S., Tappert, C., Ribeiro, T., Honeycutt, R.K., Hoard, D.W., Saar, S., 2010, ApJ 721, 1714 doi:10.1088/0004-637X/721/2/1714

[15] König, M., Beuermann, K., Gänsicke, B., 2006, A\&A 449, 1129

[16] Mauche, C.W., Liedahl, D.A., Fournier, K.B., 2003, ApJ 588, L101 doi:10.1086/375684

[17] Mauche, C.W., 2009, ApJ 706, 130 doi:10.1088/0004-637X/706/1/130

[18] Reinsch, K., Kim, Y., Beuermann, K., 2006, A\&A 457,1043

[19] Ritter, H., Kolb, U., 2003, A\&A 404, 301 (update RKcat7.20, 2013)

[20] Schwarz, R., Reinsch, K., Beuermann, K., Burwitz, V., 2005, A\&A 442,271

[21] Schwope, A., Staude, A., Vogel, J., Schwarz, R., 2004, AN, 325, 197

[22] Thomas, H.-C., Beuermann, K., Reinsch, K., Schwope, A.D., Burwitz, V., 2012, A\&A 546, A104

[23] Warner, B., 1995, Cataclysmic Variable Stars, Cambridge University Press

\section{DISCUSSION}

DAVID BUCKLEY: Would you care to comment on the fact that a certain fraction of polars have no directly (observed) determined magnetic fields (e.g. from either detection of cyclotron features or spectropolarimetry) and have been suggested to be low-field systems.

KLAUS REINSCH: The magnetic field of polars must be strong enough that spin and orbital motion of the white dwarf will be synchronized. Direct measurements of magnetic fields in polars require the systems to be in a suitable state that either cylcotron or Zeeman features can be detected. Therefore, there are still many polars lacking field determinations.

AUGUSTIN SKOPAL: How does the SED look like with the unabsorbed supersoft X-rays and dereddened far-UV fluxes? Does your unabsorbed X-ray model fit the far-UV fluxes?

KLAUS REINSCH: The "source spectrum" of the $\mathrm{X}$-ray components corrected for interstellar absorption is included as the dashed curve in Fig. 2. It is two orders of magnitude below the observed flux in the UV.

RAYMUNDO BAPTISTA: You showed Doppler tomograms of $\mathrm{H} \alpha$ emission from AM Her in low state by Kafka et al. (2010), with side emission apparently from L4 and L5 regions. An alternative explanation for that is Zeeman-Doppler spliting produced by the magnetic field of the donor star, of the order of a few $\mathrm{kG}$.

KLAUS REINSCH: Yes, I agree.

LINDA SCHMIDTOBREICK's Comment: You showed the prominences detected by Stella Kafka in AM Her. We detected the same structure in the low state of BB Dor which is a non-magnetic VY Scl system. So we think it unlikely that the magnetic field of the WD has an influence on this structure and it should rather be explained by a combination of the magnetic field of the secondary plus the Roche potential.

KLAUS REINSCH: This is indeed interesting to note. In the final remarks of their paper Kafka et al. (2010) state that prominence-like structures are likely present in disk CVs above the period gap as well. But their stability and evolution with time is expected to be different. 